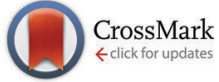

Cite this: Phys. Chem. Chem. Phys., 2016, 18, 23089

Received 21st July 2016, Accepted 1st August 2016 DOI: $10.1039 / c 6 c p 05071 a$

www.rsc.org/pccp

\section{Mutagenic induction of an ultra-fast water-chain proton wire $\dagger$}

\author{
Vered Wineman-Fisher, ${ }^{\text {ab }}$ Ron Simkovich, ${ }^{c}$ Dan Huppert, ${ }^{c}$ Kristina Trujillo, ${ }^{d}$ \\ S. James Remington ${ }^{d}$ and Yifat Miller*ab
}

\section{Introduction}

In many proteins, proton transfers occur within predefined "proton wires", and are essential to numerous important biochemical processes. $^{1-7}$ Typically, the proton wire involves well defined, precisely arranged amino acid side chains 1-7 while in other cases, the proton wire involves water-side chain hydrogen bonds that are presumably less well organized. ${ }^{8-11}$ Despite the innumerable documented examples of proton transfers that occur via amino acid side chains, and a handful that involve water-chains, ${ }^{8-11}$ it has yet to be demonstrated that an amino-acid based proton wire can be replaced by a functional water-chain wire.

We herein use the green fluorescent protein (GFP) in an un-precedent fashion as an ultra-fast probe for investigating the activity of a mutagenically induced "water-chain" proton wire. This is achieved by replacing the hydroxyl group of the hydrophilic side chain Ser205 by the hydrogen atom of the smallest side chain Gly, i.e. S205G. This replacement led to a cavity in the proton wire domain that is then now occupied by water molecules from the interior $\beta$-barrel of the GFP. These water molecules function as an ultra-fast water chain in the proton wire domain (Fig. 1).

During fluorescence emission, wild-type (wt)-GFP exhibits well documented ultrafast, excited state proton transfer. A proton is

\footnotetext{
${ }^{a}$ Department of Chemistry, Ben-Gurion University of the Negev, P.O. Box 653, Be'er Sheva 84105, Israel. E-mail: ymiller@bgu.ac.il; Fax: +972-86428709; Tel: $+972-86428705$

${ }^{b}$ Ilse Katz Institute for Nanoscale Science and Technology,

Ben-Gurion University of the Negev, Beér-Sheva 84105, Israel

${ }^{c}$ Raymond and Beverly Sackler Faculty of Exact Sciences, School of Chemistry,

Tel Aviv University, Tel Aviv 69978, Israel

${ }^{d}$ Department of Physics and Institute of Molecular Biology, University of Oregon, Eugene, USA

$\dagger$ Electronic supplementary information (ESI) available. See DOI: 10.1039/c6cp05071a
}

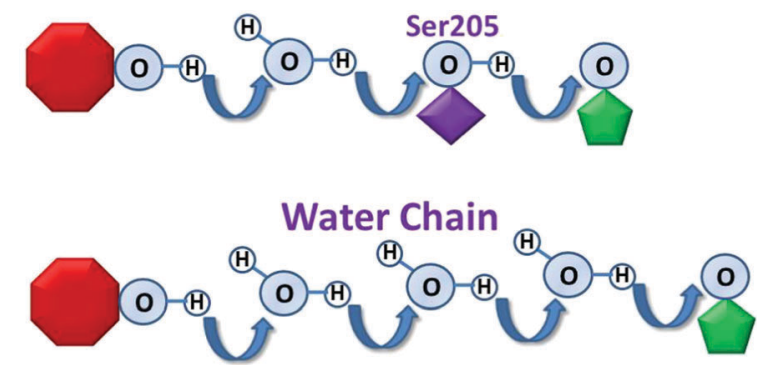

Fig. 1 The replacement of the hydroxyl group of the hydrophilic sidechain Ser205 (top) by $\mathrm{H}$ atom of the smallest sidechain Gly led to waterchain proton wire (bottom). Top: wt-GFP proton wire. Bottom: The new "engineered" proton wire. The chromophore is colored in red and Glu222 is colored in green.

transported within several ps (biphasic, rates $\sim 3$ and $\sim 12 \mathrm{ps})^{12}$ from the excited state of the chromophore via a bound water molecule and Ser205 to the acceptor, a carboxylate oxygen of Glu222 (Fig. 1). ${ }^{13-18}$ Ultrafast spectroscopy, combined with crystal structure and molecular dynamics analyses in wild type and mutant GFP allows us to alter and measure proton transfer rates, leading to a deeper understanding of the mechanisms of proton transfer. ${ }^{4,19-28}$

Mutagenesis of GFP in the vicinity of the proton wire domain, such as replacement of the hydroxyl group of Ser205 (Fig. 1) and or the side chain of Thr203 by a hydrophobic isopropyl or methyl group (Val or Ala) resulted in substantial (up to 30-fold) reduction of proton transfer rates. ${ }^{4,17,28}$ On the other hand, simultaneous replacement of Ser205 by Ala and Thr203 by Val had a different result: a rearranged proton wire 21 which involved water molecules infrequently entering from the bulk, leading to a 350-fold reduction of the proton transfer rate compared to wt-GFP. However the general trend is that replacements of hydrophilic side chains by hydrophobic side 
chains dramatically decreases the proton transfer rates. This led us to investigate a different approach, namely to remove the intermediate side chain altogether. The resultant cavity is occupied by water molecules, which we herein show function as an ultrafast water-chain proton wire.

\section{Materials and methods}

\section{Mutagenesis, protein expression, and crystallization}

The S205G mutation was introduced into GFP and protein was prepared as previously described for the mutant $\mathrm{S} 205 \mathrm{~V}^{22}$ Mutations were verified by sequencing the entire gene. Rectangular prismatic crystals of S205G were obtained in 2 days by hanging drop vapor diffusion. Crystallization setups consisted of $3 \mu \mathrm{l}$ drops containing one part protein solution $(\mathrm{A} 280=18.0)$ in $50 \mathrm{mM}$ Hepes, pH 7.9, 0.3 M NaCl and one part well solution. Crystals of S205G grew best against well solutions of $2.0 \mathrm{M}$ malic acid, $\mathrm{pH}$ 8.0, $0.1 \mathrm{M}$ Tris, with a $10 \%$ drop volume additive of $60 \mathrm{mM}$ detergent ( $n$-nonyl- $\beta$-D-maltoside).

\section{Data collection and structure solution}

X-ray diffraction data were collected at $100 \mathrm{~K}$ to 2.49 A resolution (limited by the crystalline order) using the in house Rigaku Raxis-IV. Diffraction images were integrated and the data were scaled using the HKL program suite (Table S2, ESI $\dagger$ ). The structure of S205G was solved by molecular replacement using the EPMR program ${ }^{29}$ with WT-GFP (RCSB Protein Data Bank ID 1EMB) as a search model. The model was refined with the CCP4 suite of crystallographic programs ${ }^{30}$ Manual model building was conducted with the Coot program. ${ }^{31}$

There are two independent but structurally very similar molecules of S205G in the asymmetric unit, with one molecule of malate (Fig. S1 and S2, ESI $\dagger$ ) bound adjacent to the chromophore within each monomer. In each case, the side chain of Tyr145 swings out about 45 degrees, and the main chain at the site of the mutation 205 moves outward toward solvent to accommodate the bound malate molecule (Fig. S2, ESI $\dagger$ ). A single molecule of the detergent $n$-nonyl- $\beta$-D-maltoside was identified, bound to a crevice at the interface between the two molecules in the asymmetric unit. Final model statistics are satisfactory and are presented in Table S2 (ESI $\dagger$ ).

\section{Steady state spectroscopy}

The steady-state absorption spectra were recorded by a Horiba Jobin Yvon FluoroMax-3 spectrofluorometer.

\section{Time-resolved emission spectroscopy}

The fluorescence up-conversion technique was employed in this study to measure the time-resolved emission of wt-GFP and S205G GFP mutant at room temperature. The laser used for the fluorescence up-conversion was a cavity-dumped Ti:sapphire femtosecond laser, (Mira, Coherent), which provides short, $150 \mathrm{fs}$, pulses at about $800 \mathrm{~nm}$. The cavity dumper operated with a relatively low repetition rate of $800 \mathrm{kHz}$. The up-conversion system (FOG-100, CDP, Russia) operated at $800 \mathrm{kHz}$. The samples were excited by pulses of $\sim 8 \mathrm{~mW}$ on average at the SHG frequency.
The time response of the up-conversion system is evaluated by measuring the relatively strong Raman-Stokes line of water shifted by $3600 \mathrm{~cm}^{-1}$. It was found that the Full width at half maximum (fwhm) of the signal is $250 \mathrm{fs}$. Samples were placed in a rotating optical cell to avoid degradation. We found that, during our five-minute time-resolved measurements in a cell rotating at a frequency of $10 \mathrm{~Hz}$, the degradation of the sample was marginal, and had no effect on the signal's decay profile. Experiments were carried out on samples of concentrations of about $1 \mathrm{mM}$ or less.

\section{Kinetic isotope effect (KIE) - estimation of rates}

The kinetic isotope effect (KIE) is defined as the ratio of rate constants with and without isotopic substitution. The kinetic isotope effect is a fingerprint of an ESPT process and is found in all hydroxyaryl photoacids. In ESPT to water, the dissociation rate coefficient ratio in $\mathrm{H}_{2} \mathrm{O}$ and $\mathrm{D}_{2} \mathrm{O}$ is $k^{\mathrm{H}} / k^{\mathrm{D}}$, which is the value we use here and in the main manuscript. ${ }^{32,33}$ We use the spherical symmetric diffusion program (SSDP) developed by Krissnel and Agmon in order to fit the experimental results, and calculate $k^{\mathrm{D}}$ or $k^{\mathrm{H}}$, and derive the KIE. ${ }^{34}$

For wt-GFP the KIE is rather large, KIE $\sim 5$. In bulk aqueous solution weak photoacids with $\mathrm{p} K_{\mathrm{a}}{ }^{*}>0$ exhibits a KIE of about 3. It is smaller for very strong photoacids with $\mathrm{p} K_{\mathrm{a}}{ }^{*}>0-2$. Fig. S3 $(\mathrm{ESI} \dagger)$ shows the normalized steady state fluorescence spectrum of $\mathrm{S} 205 \mathrm{G}$ in $\mathrm{H}_{2} \mathrm{O}$ and $\mathrm{D}_{2} \mathrm{O}$ on a semilogarithmic scale. The intensity ratio between the $\mathrm{ROH}$ band at $450 \mathrm{~nm}$ and the $\mathrm{RO}^{-}$ band at $510 \mathrm{~nm}$ differs by a factor of 3 in $\mathrm{D}_{2} \mathrm{O}$ when compared with that obtained in $\mathrm{H}_{2} \mathrm{O}$. This factor is approximately the kinetic isotope effect expected for an ESPT process to water.

Fig. S4 (ESI $\dagger$ ) shows the time-resolved fluorescence of the $\mathrm{ROH}$ form of S205G mutant measured at $460 \mathrm{~nm}$ in both $\mathrm{H}_{2} \mathrm{O}$ and $\mathrm{D}_{2} \mathrm{O}$ solutions. As seen in the figure the fluorescence decay of the $\mathrm{D}_{2} \mathrm{O}$ sample is rather slower than that of the $\mathrm{H}_{2} \mathrm{O}$ sample. Fig. S5 (ESI $\dagger$ ) shows the time-resolved emission of the $\mathrm{RO}^{-}$ form of S205G mutant measured at $520 \mathrm{~nm}$ in both $\mathrm{H}_{2} \mathrm{O}$ and $\mathrm{D}_{2} \mathrm{O}$ samples. As expected, the rise of the signal of the $\mathrm{D}_{2} \mathrm{O}$ sample is much longer than that of the $\mathrm{RO}^{-} \mathrm{S} 205 \mathrm{G}$ mutant signal in $\mathrm{H}_{2} \mathrm{O}$.

\section{Examining the role of the malate in the ESPT}

We examined whether malate plays a role in the ESPT process. To this end we compared the spectroscopy measurements of the GFP S205G mutant without the malate to those of the wt-GFP. Time resolved emission spectra were obtained for S205G in solutions with varying concentrations of malate and compared to results obtained in the absence of malate. Fig. S6 and S7 (ESI $\dagger$ ) illustrate that the time-resolved emission spectra of the $\mathrm{ROH}$ and the $\mathrm{RO}^{-}$forms of $\mathrm{S} 205 \mathrm{G}$ are similar with or without malate at various concentrations, up to $3 \mathrm{M}$ of malate. Furthermore, we found that the malate does not affect the fluorescence decay of the $\mathrm{ROH}$ form of the mutant up to a concentration of $1 \mathrm{M}$ of malate (Fig. S7, ESI $\dagger$ ), also at longer times (Fig. S8, ESI $\dagger$ ). Finally, the steady state excitation and emission spectra of $\mathrm{S} 205 \mathrm{G}$ in the presence of malate demonstrates similar bands to those obtained in the absence of malate (Fig. S9, ESI $\dagger$ ). These results 
led us to conclude that the malate does not play a role in the ESPT process (at least up to $3 \mathrm{M}$ ), although the malate is near the chromophore and forms hydrogen bonds as observed from the crystal structure.

\section{Weller's formulation}

Following Weller's formulation: ${ }^{35}$

$$
k_{\mathrm{PT}} \cong\left(I_{\mathrm{F}}^{\mathrm{RO}^{-}} / I_{\mathrm{F}}^{\mathrm{ROH}}\right) \cdot \tau_{\mathrm{F}}^{\mathrm{RO}^{--1}}
$$

This equation neglects proton geminate recombination and assumes the same radiative life-time for both $\mathrm{ROH}$ and $\mathrm{RO}^{-}$.

\section{Molecular dynamics (MD) simulations protocol}

Molecular dynamics (MD) simulations were performed starting from the 5HZO Protein Data Bank entry for the S205G mutant of GFP. MD simulations of the solvated structures were performed in the NPT ensemble using the NAMD ${ }^{36}$ with the CHARMM27 force field. ${ }^{37}$ The structures were energy minimized and explicitly solvated in a TIP3P water box ${ }^{38,39}$ with a minimum distance of $15 \AA$ A from each edge of the box. Each water molecule within $2.5 \AA$ of the protein was removed. Counter ions were added at random locations to neutralize the protein's charge. The Langevin piston $\operatorname{method}^{40,41}$ with a decay period of $100 \mathrm{fs}$ and a damping time of 50 fs was used to maintain a constant pressure of $1 \mathrm{~atm}$. The temperature $310 \mathrm{~K}$ was controlled by a Langevin thermostat with a damping coefficient of 10 ps. The short-range van der Waals (VDW) interactions were calculated using the switching function, with a twin range cutoff of 10.0 and 12.0 A. Long-range electrostatic interactions were calculated using the particle mesh Ewald method with a cutoff of $12.0 \AA^{42,43}$ The equations of motion were integrated using the leapfrog integrator with a step of $1 \mathrm{fs}$. The counter ions and water molecules were allowed to move. The minimized solvated system was energy minimized for 5000 additional conjugate gradient steps and 20000 heating steps at $250 \mathrm{~K}$, with all atoms allowed to move. Then, the system was heated from 250 to $310 \mathrm{~K}$ for $300 \mathrm{ps}$ and equilibrated at $310 \mathrm{~K}$ for $300 \mathrm{ps}$. The simulation ran for $60 \mathrm{~ns}$, as we previously performed for wt-GFP and other mutants. ${ }^{17,28}$ To examine whether the timescale of $60 \mathrm{~ns}$ of simulations is a reasonable timescale, we examined the root-mean square deviations (RMSDs) of the system along the time of the simulations (Fig. S10, ESI $\dagger$ ). One can see from Fig. S10 (ESI $\dagger$ ) that the system has been converged; therefore, these conditions (60 ns and $310 \mathrm{~K}$ ) were carefully chosen for such systems.

\section{Results and discussion}

\section{Crystal structure of GFP S205G}

The crystal structure of the S205G mutant was determined to 2.5 Å resolution using crystals grown using sodium malate as the precipitant (SI text, PDB ID code: 5HZO). There are two independent but nearly identical protein molecules in the asymmetric unit. In each molecule, a significant conformational change involving Tyr145 opened a cavity adjacent to the chromophore, which was observed to contain a strong electron density
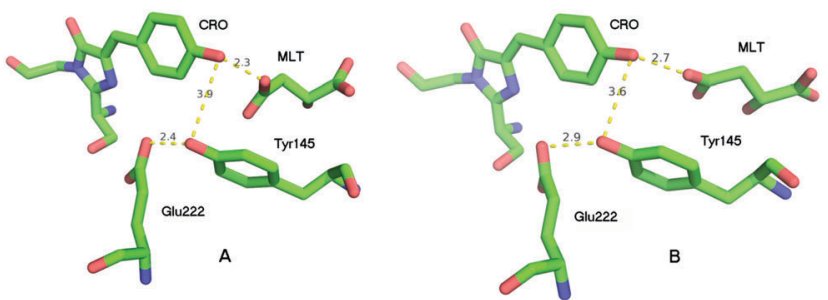

Fig. 2 The proton wire domains of GFP S205G as observed from the crystal structure.

feature unconnected to the protein. The features were adequately modeled as malate (Fig. S1 and S2, ESI $\dagger$ ), however the malate molecules are not in a position to participate in the ESPT process, and there are no obvious candidates (including well-ordered water molecules) to substitute for the proposed functional role of the Ser205 hydroxyl group in wt-GFP.

The overall crystal structure of the GFP S205G mutant is very similar to that previously described for wt-GFP, however, structural changes near the site of the mutation are clearly revealed by the electron density map. Fig. 2 shows excerpts from the A and $\mathrm{B}$ molecules in the asymmetric unit, and illustrates the chromophore cavity containing the proton wire region. In each case the presumed malate molecule is near the chromophore (CRO), with one oxygen making a presumed hydrogen bond
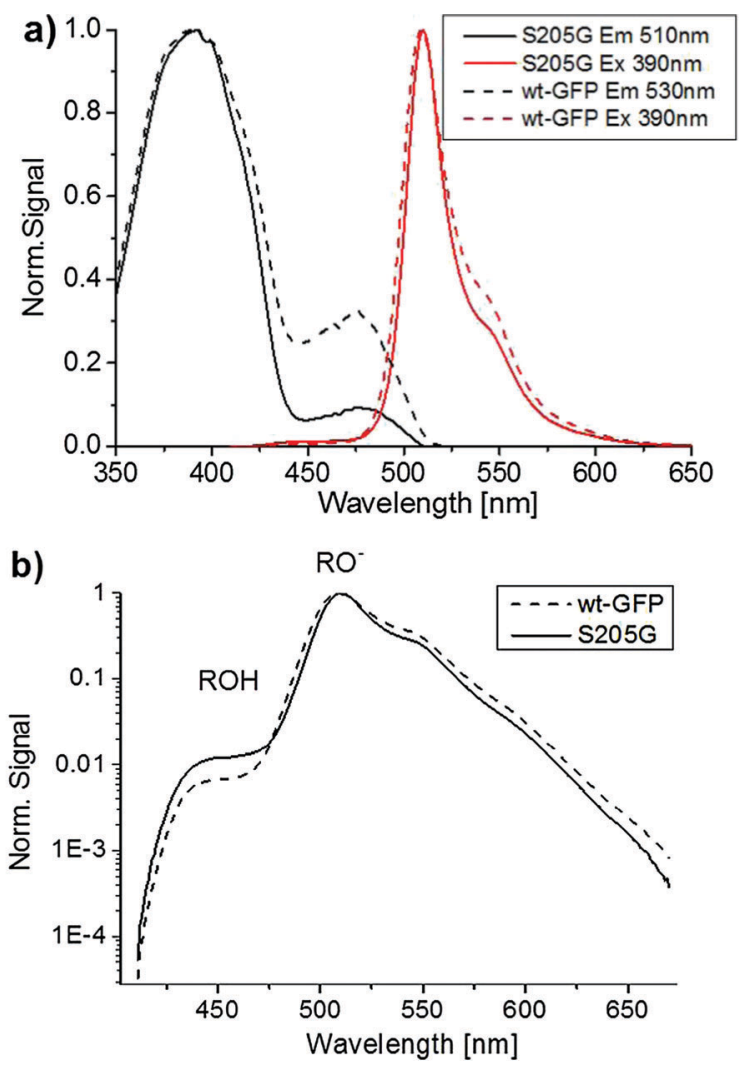

Fig. 3 Steady state excitation and emission spectra of (a) S205G mutant (solid line) and wt-GFP (dashed line) in HEPES pH $\sim 7$ buffer solution, shown on a linear scale (b) steady state emission spectra of (a) S205G mutant (solid line) and wt-GFP (dashed line) in HEPES pH $\sim 7$ buffer. 
with the chromophore hydroxyl, but not in a position to participate in ESPT to Glu222. While it is conceivable that the hydroxyl of Tyr145 could replace the functionality of the Ser205 hydroxyl in wt-GFP, the orientation is very poor and the distance between the CRO and the Tyr145 hydroxyl is too large for direct proton transfer.

The proton transfer process in GFP S205G is dramatically faster than other mutants and has closer rate to the wt-GFP

Time-resolved emission spectroscopy, ultrafast fluorescence up-conversion spectroscopy and steady-state excitation and emission spectroscopy have been applied to investigate the proton transfer rates in the GFP S205G with and without sodium malate in solution. Interestingly, these spectroscopic measurements demonstrated similar proton transfer rates, therefore indicating that malate has no detectable effect on the excited-state proton transfer (ESPT) process (Fig. S3-S7, ESI $\dagger$ ).

Fig. S5 (ESI $\dagger$ ) shows the steady state excitation and emission spectra of the GFP S205G mutant. One can see that the fluorescence spectrum consists of a weak emission band of the $\mathrm{ROH}$ and a strong emission band of $\mathrm{RO}^{-}$. These results indicate an efficient ESPT process of the protonated chromophore in the S205G mutant. Time-resolved fluorescence of the mutant supports the assignment of an ESPT process of the excited chromophore and that the proton transfer to amino acids via a proton acceptor, which is one or more water molecules within the $\beta$-barrel of the GFP (Fig. S11, ESI $\dagger$ ). Fig. 3a compares the steady state excitation and emission spectra of GFP S205G mutant and the wt-GFP. The excitation spectrum of the wt-GFP shows that the relative absorbance of the $\mathrm{ROH}$ and $\mathrm{RO}^{-}$forms in the ground state are $75 \%$ and $25 \%$, respectively, while for the GFP S205G mutant the relative absorbance of $\mathrm{ROH}$ and $\mathrm{RO}^{-}$forms are $91 \%$ and $9 \%$, respectively. The excitation spectrum of GFP S205G mutant shows a weaker intensity for $\mathrm{RO}^{-}$at $\mathrm{pH} \sim 7$, therefore the $\mathrm{p} K_{\mathrm{a}}$ of the mutant is shifted to higher wavelengths. The steady state emission spectra of both wt-GFP and the mutant shows that the $\mathrm{ROH}$ emission band intensity is two orders of magnitude weaker than that of the $\mathrm{RO}^{-}$ emission (Fig. 3b).

Furthermore, the ROH emission intensity of the wt-GFP is weaker than the mutant and therefore one can expect that the ESPT rate of the wt-GFP is faster than that of the mutant. To estimate the ESPT rate we applied Weller's formulation ${ }^{35}$ (ESI $\dagger$ text). For an emission life time of the $\mathrm{RO}^{-}=3 \mathrm{~ns}$, the estimated ESPT rate constants are $5.1 \times 10^{10} \mathrm{~s}^{-1}$ and $2.7 \times 10^{10} \mathrm{~s}^{-1}$ for the wt-GFP and the GFP S205G, respectively. Therefore, the ESPT rate of the GFP S205G is about twice slower than that of the wt-GFP. The time-resolved emission spectra of the $\mathrm{ROH}$ and the $\mathrm{RO}^{-}$forms of both the wt-GFP and the mutant are seen in Fig. 4. Table S1 (ESI $\dagger$ ) provides the fitting parameters of the three-exponential fitting function to the $\mathrm{ROH}$ fluorescence decay of the wt-GFP and the mutant. The average decay time
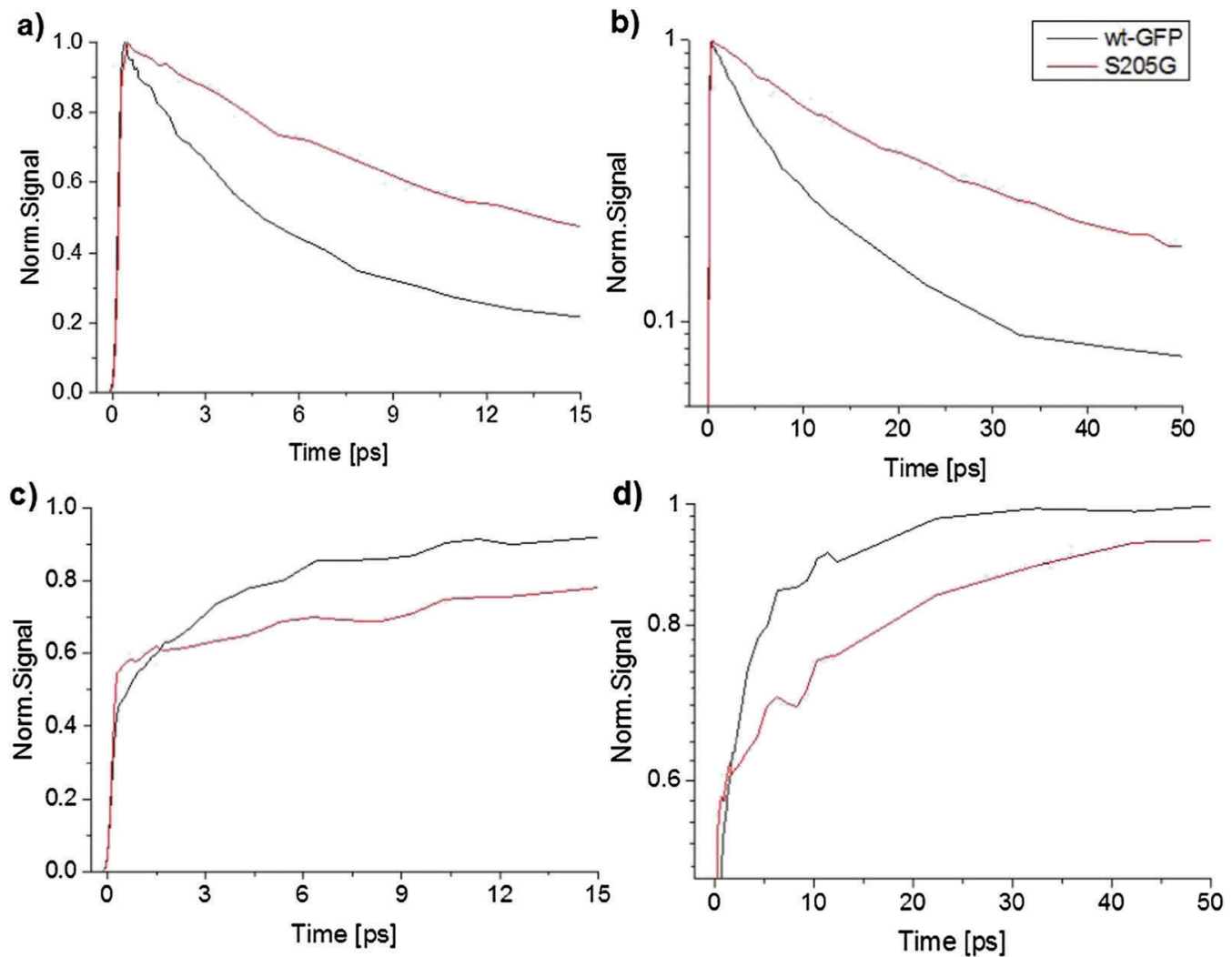

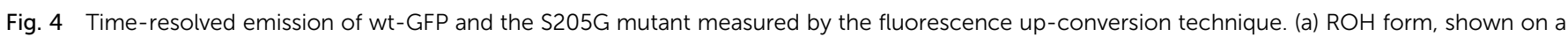

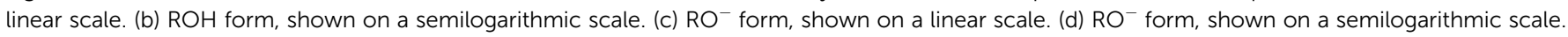




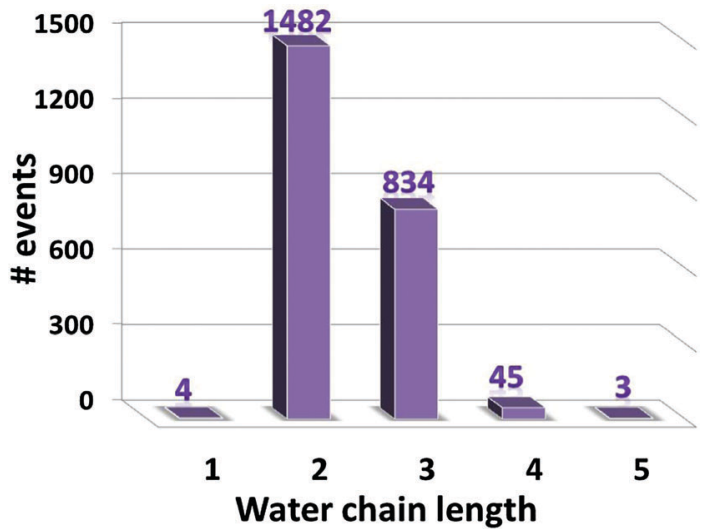

Fig. 5 The distribution events of the number of water molecules that participate as water chain in the "proton wire" pathway.

of the ROH of the mutant is three times longer than that of the wt-GFP (Fig. 4a and b), the long rise-time component that is assigned to the formation of $\mathrm{RO}^{-}$due to the ESPT process shows a longer time for the mutant compared to the wt-GFP (Fig. $4 \mathrm{c}$ and d). This indicates that the ESPT rate of the mutant is three times slower than for the wt-GFP. The ESPT rate has a similar value to that observed for an ESPT from a weak photoacid to acetate ion via a few water molecules. ${ }^{44}$ Therefore, the estimated ESPT rate study suggests that the proton wire study involves water molecules (Fig. S3, ESI $\dagger$ ). We also measured the kinetic isotopic effect (KIE) of the mutant and compared it with the KIE of the wt-GFP (ESI $\dagger$ text, Fig. S4 and S12). The KIE value of wt-GFP is around 5 and for the mutant it is $\sim 3.5$. The lower KIE of S205G is similar to the ESPT study of many photoacids to bulk water and therefore suggests that the ESPT process involves water molecules.

\section{Two novel "proton-wire" pathways in the S205G GFP involve} water molecules

To provide insight into the ESPT mechanism in the mutant, we performed all-atom explicit MD simulations. The details for the procedure of MD simulations are reported and illustrated in the ESI $\uparrow$. For the MD simulations we used the 5HZO Protein Data Bank entry for the S205G mutant of GFP. Starting the MD simulations from the S205G crystal structure, the proton wire that is seen in Fig. 2 has changed during the simulation: the position of Tyr145 fluctuated during the simulations and the distance between the Tyr145 and the CRO increased (Fig. S13, ESI $\dagger$ ). This may explain the relatively large $\mathrm{O}-\mathrm{O}$ distance $(3.9 \AA)$ between the TYR145 and the CRO that is observed from the crystal structure (Fig. 2). However, a new proton wire is obtained from the MD simulations at a relatively short time and remains stable until the end of the simulations. Interestingly, the new structure of the mutant shows that the Tyr145 fluctuates and forms a "hole" and thus allows water molecules to accommodate this "hole" (Fig. S14, ESI $\uparrow$ ). The Tyr145 fluctuated also between the CRO and the Glu222 (Fig. S15, ESI†). When the Tyr145 is far from the Glu222 it allows water molecules to accommodate the "hole" and consequently the Glu222 is more solvated
(Fig. S16, ESI $\dagger$ ). We therefore propose that the water molecules that accommodate this "hole" participate in the proton wire. To estimate the number of water molecules that participate in the proton wire, we computed the event distributions of water molecules that form a proton wire between the CRO and the Glu222. Our statistical calculations have shown that it is more likely that 2-3 water molecules participate in the proton wire (Fig. 5). Following the MD simulations we propose two novel "proton-wire" pathways, as one can see from the MD simulation snapshots (Fig. 6). The first pathway involves the phenol group of the chromophore, a water molecule, Typ145 and Glu222 or the phenol group of the chromophore, Typ145, a water molecule and Glu222 (Fig. 6a). This pathway is less frequently seen along the MD simulations (Fig. S15, ESI $\dagger$ ). The second pathway involves the phenol group of the chromophore, 2-3 water molecules and Glu222 (Fig. 6b). This pathway is more frequent along the MD simulations. One can see from the MD simulations that these two pathways may be concerted simultaneously (Fig. 5a), i.e. may occur simultaneously and therefore we suggest that they may compete, but according to the frequent snapshots seen along the MD simulations, the Tyr145 is less involved in the proton wire (Fig. S15, ESI $\dagger$ ).

In summary, the MD simulations proposed two new possible proton transfer pathways, in which one pathway involves only one water molecule and the second involved 2-3 water molecules.

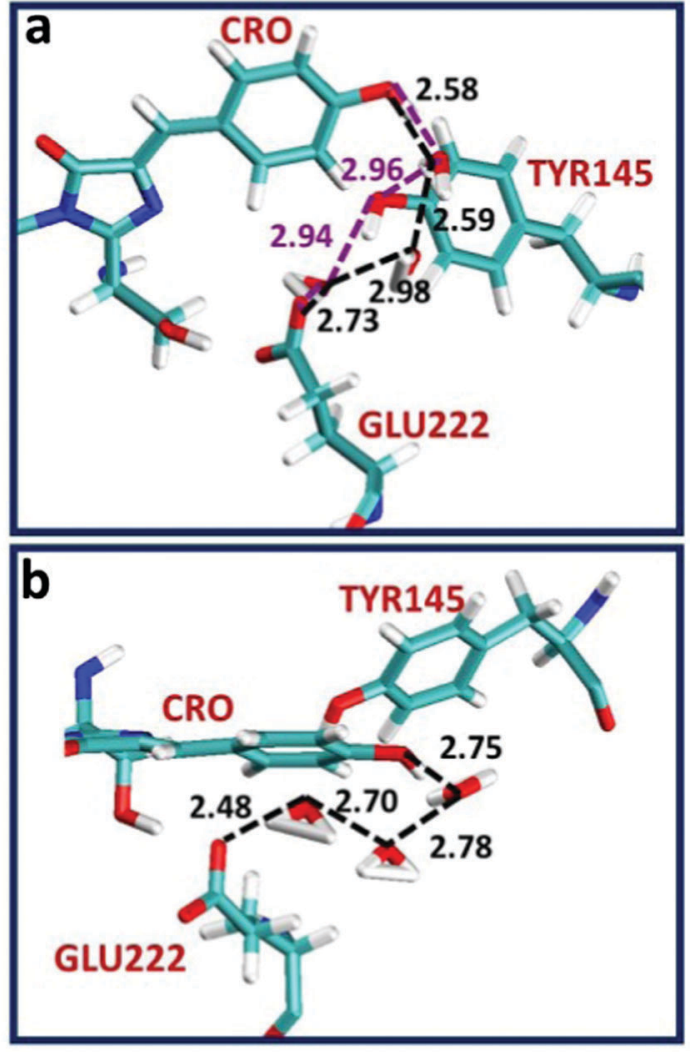

Fig. 6 Two novel "proton-wire" pathways in the S205G GFP obtained from the MD simulations consist of water chain proton wire. The "proton wire" pathway seen in (b) is more frequent than the pathway seen in (a). 
In contrast to our previous study, in which we have shown that the water molecules involved in the proton wire process are infrequently entering from the bulk in the GFP double mutant T203V/S205A and with a rate of $\sim 350$-fold reduction compare to wt-GFP, in the new GFP S205G mutant the water molecules are internal, i.e. in the $\beta$-barrel, and the rate is only $\sim 20 \mathrm{ps}$.

\section{Conclusions}

To advance our mechanistic understanding of ESPT processes in GFP, we present a crystal structure and time resolved spectroscopic analysis of the S205G mutant. The S205G mutation was introduced to study the role of the hydroxyl group in the proton transfer reaction. However the mutation introduced flexibility into the protein, leading to structural changes that create a binding site for malate (used as a precipitant for crystallization purposes) which interacts with the chromophore. It seems likely that this mutation could lead to a new class of indicator proteins, with binding sites engineered to accommodate various small molecules of interest. The crystal structure revealed no candidate side chains that could replace the role of the hydroxyl of Ser205 in ESPT. However, examining the ESPT of the GFP S205G mutant using a variety of optical techniques have revealed two interesting results: first, the GFP S205G demonstrated ESPT. Second, the kinetics of the ESPT process showed a fast process with comparison to the previous GFP mutants, such as T203V/S205V. To investigate the molecule mechanism of ESPT in the mutant, we performed all-atom explicit MD simulations. The simulations allow following the dynamics of the GFP S205G mutant in solvated water molecules. Interestingly, during the MD simulations Tyr145 fluctuated (in agreement with the crystal structure), opening a "hole" between the chromophore and Glu222 and allowing entry of water molecules from the interior of the $\beta$-barrel to participate simultaneously in two competing novel proton wire mechanisms. In this study, the unique fluorescence properties of the GFP protein are used as an ultra-fast probe for testing the activity of a mutagenically induced water-chain proton wire. Namely, re-placement of the hydroxyl group of a hydrophilic side chain in the wt-GFP proton wire by an approx. $5 \AA$ cavity induces formation of a water-chain that functions as a proton wire with response times within $10 \mathrm{ps}$ of the wild type protein. We understand this remarkable rate retention as a natural consequence of the inherent rate Grotthuss mechanism proton transfer in water.

\section{Acknowledgements}

This project is funded by the FP7-PEOPLE-2011-CIG (YM; CIG 303741) and in part supported by Grant No. 2011/128 from the United States-Israel Binational Science Foundation (BSF). DH and SJR were funded by the BSF. SJR acknowledges support from the National Science Foundation, grant MCB-1021374. All simulations were performed using the high-performance computational facilities of the Miller lab in the BGU HPC computational center. The support of the BGU HPC computational center staff is greatly appreciated.

\section{References}

1 R. A. Frank, C. M. Titman, J. V. Pratap, B. F. Luisi and R. N. Perham, Science, 2004, 306, 872.

2 W. Han, R. C. Cheng, M. C. Maduke and E. Tajkhorshid, Proc. Natl. Acad. Sci. U. S. A., 2014, 111, 1819.

3 A. J. Hughes, A. M. Tentori and A. E. Herr, J. Am. Chem. Soc., 2012, 134, 17582.

4 S. P. Laptenok, A. Lukacs, A. Gil, R. Brust, I. V. Sazanovich, G. M. Greetham, P. J. Tonge and S. R. Meech, Angew. Chem., Int. Ed. Engl., 2015, 54, 9303.

5 Y. Nishihara and A. Kitao, Proc. Natl. Acad. Sci. U. S. A., 2015, $112,7737$.

6 U. S. Raghavender, S. Aravinda, N. Shamala, Kantharaju, R. Rai and P. Balaram, J. Am. Chem. Soc., 2009, 131, 15130.

7 A. Shinobu, G. J. Palm, A. J. Schierbeek and N. Agmon, J. Am. Chem. Soc., 2010, 132, 11093.

8 M. Barboiu and A. Gilles, Acc. Chem. Res., 2013, 46, 2814.

9 E. Freier, S. Wolf and K. Gerwert, Proc. Natl. Acad. Sci. U. S. A., 2011, 108, 11435.

10 E. Persson and B. Halle, J. Am. Chem. Soc., 2008, 130, 1774.

11 H. Yu and S. W. Rick, J. Am. Chem. Soc., 2009, 131, 6608.

12 M. Chattoraj, B. A. King, G. U. Bublitz and S. G. Boxer, Proc. Natl. Acad. Sci. U. S. A., 1996, 93, 8362.

13 T. M. Creemers, A. J. Lock, V. V. Subramaniam, T. M. Jovin and S. Volker, Nat. Struct. Biol., 1999, 6, 706.

14 J. N. Henderson, R. Gepshtein, J. R. Heenan, K. Kallio, D. Huppert and S. J. Remington, J. Am. Chem. Soc., 2009, 131, 4176.

15 C. C. Hsieh, P. T. Chou, C. W. Shih, W. T. Chuang, M. W. Chung, J. Lee and T. Joo, J. Am. Chem. Soc., 2011, 133, 2932.

16 Y. Ma, Q. Sun, Z. Li, J. G. Yu and S. C. Smith, J. Phys. Chem. B, 2012, 116, 1426.

17 R. Simkovitch, A. Huppert, D. Huppert, S. J. Remington and Y. Miller, J. Phys. Chem. B, 2013, 117, 11921.

18 J. J. van Thor, T. Gensch, K. J. Hellingwerf and L. N. Johnson, Nat. Struct. Biol., 2002, 9, 37.

19 Y. Erez, R. Gepshtein, I. Presiado, K. Trujillo, K. Kallio, S. J. Remington and D. Huppert, J. Phys. Chem. B, 2011, 115, 11776.

20 C. Fang, R. R. Frontiera, R. Tran and R. A. Mathies, Nature, 2009, 462, 200.

21 M. A. Lill and V. Helms, Proc. Natl. Acad. Sci. U. S. A., 2002, 99, 2778.

22 X. Shu, P. Leiderman, R. Gepshtein, N. R. Smith, K. Kallio, D. Huppert and S. J. Remington, Protein Sci., 2007, 16, 2703.

23 D. Stoner-Ma, A. A. Jaye, K. L. Ronayne, J. Nappa, S. R. Meech and P. J. Tonge, J. Am. Chem. Soc., 2008, 130, 1227.

24 D. Stoner-Ma, E. H. Melief, J. Nappa, K. L. Ronayne, P. J. Tonge and S. R. Meech, J. Phys. Chem. B, 2006, 110, 22009.

25 J. J. van Thor, K. L. Ronayne, M. Towrie and J. T. Sage, Biophys. J., 2008, 95, 1902.

26 O. Vendrell, R. Gelabert, M. Moreno and J. M. Lluch, J. Am. Chem. Soc., 2006, 128, 3564.

27 O. Vendrell, R. Gelabert, M. Moreno and J. M. Lluch, J. Chem. Theory Comput., 2008, 4, 1138. 
28 V. Wineman-Fisher, R. Simkovitch, S. Shomer, R. Gepshtein, D. Huppert, M. Saif, K. Kallio, S. J. Remington and Y. Miller, Phys. Chem. Chem. Phys., 2014, 16, 11196.

29 C. R. Kissinger, D. K. Gehlhaar and D. B. Fogel, Acta Crystallogr., Sect. D: Biol. Crystallogr., 1999, 55, 484.

30 The CCP4 suite: programs for protein crystallography, Acta Crystallogr., Sect. D: Biol. Crystallogr., 1994, 50, 760-763.

31 P. Emsley and K. Cowtan, Acta Crystallogr., Sect. D: Biol. Crystallogr., 2004, 60, 2126.

32 N. Agmon, J. Phys. Chem. A, 2005, 109, 13.

33 A. L. Kohen and H. H. Limbach, The Kinetic Isotope Effect in the Photo-Dissociation Reaction of Excited-State Acids in Aqueous Solutions, Isotope effects in chemistry and biology, CRC Press, 2005.

34 E. B. Krissinel and N. Agmon, J. Comput. Chem., 1996, 17, 1085. 35 A. Weller, Prog. React. Kinet., 1961, 1, 187.

36 L. Kale, R. Skeel, M. Bhandarkar, R. Brunner, A. Gursoy, N. Krawetz, J. Phillips, A. Shinozaki, K. Varadarajan and K. Schulten, J. Comput. Phys., 1999, 151, 283.

37 A. D. MacKerell, D. Bashford, M. Bellott, R. L. Dunbrack, J. D. Evanseck, M. J. Field, S. Fischer, J. Gao, H. Guo, S. Ha,
D. Joseph-McCarthy, L. Kuchnir, K. Kuczera, F. T. K. Lau, C. Mattos, S. Michnick, T. Ngo, D. T. Nguyen, B. Prodhom, W. E. Reiher, B. Roux, M. Schlenkrich, J. C. Smith, R. Stote, J. Straub, M. Watanabe, J. Wiorkiewicz-Kuczera, D. Yin and M. Karplus, J. Phys. Chem. B, 1998, 102, 3586.

38 W. L. Jorgensen, J. Chandrasekhar, J. D. Madura, R. W. Impey and M. L. Klein, J. Chem. Phys., 1983, 79, 926.

39 M. W. Mahoney and W. L. Jorgensen, J. Chem. Phys., 2000, 112, 8910.

40 S. E. Feller, Y. H. Zhang, R. W. Pastor and B. R. Brooks, J. Chem. Phys., 1995, 103, 4613.

41 G. J. Martyna, D. J. Tobias and M. L. Klein, J. Chem. Phys., 1994, 101, 4177.

42 T. Darden, D. York and L. Pedersen, J. Chem. Phys., 1993, 98, 10089.

43 U. Essmann, L. Perera, M. L. Berkowitz, T. Darden, H. Lee and L. G. Pedersen, J. Chem. Phys., 1995, 103, 8577.

44 O. F. Mohammed, D. Pines, E. T. Nibbering and E. Pines, Angew. Chem., Int. Ed. Engl., 2007, 46, 1458. 